\title{
Antenatal and Neonatal Endocrinology
}

\author{
Khalid Hussain \\ Clinical and Molecular Genetics Unit, The Developmental Endocrinology Research Group, Institute of Child \\ Health, University College London, London, UK
}

\section{A: Neonatal glucose and insulin physiology}

\author{
Glucose monitoring in neonates: need for accurate and non-invasive methods \\ Woo HC, Tolosa L, El-Metwally D, Viscardi RM \\ Division of Neonatology, Department of Pediatrics, School of Medicine, University of Maryland, Baltimore, MD, USA \\ Arch Dis Child Fetal Neonatal Ed 2014;99:F153-F157
}

Background: Neonatal hypoglycemia can lead to devastating consequences. Thus, constant, accurate and safe glucose monitoring is imperative in neonatal care. However, point-of-care (POC) devices for glucose testing currently used for neonates were originally designed for adults and do not address issues specific to neonates.

Methods: This review addresses currently available monitoring options and describes a new methodology for non-invasive blood glucose monitoring in newborns.

Results: The authors outline the current blood glucose monitoring options in the newborn period and then describe a potentially novel non-invasive method for measuring blood glucose levels. The method developed depends on using a glucose sensor based on the glucose-binding protein (GBP). The GBP is found in the periplasmic space of Gram-negative bacteria. The GBP is not an enzyme and signal transduction is effected through the change in conformation of the protein upon binding of glucose. The advantage of this change in protein conformation has been taken up by strategically introducing a cysteine mutation at position 255 and labelling that mutation with the polarity-sensitive dye, acrylodan. Changes in the fluorescent properties of the dye are then correlated with the concentration of the analyte (glucose) in the sample.

Conclusions: Non-invasive blood glucose monitoring method could potentially change the way we measure bedside blood glucose in the newborn period.

\section{Novel insights into pancreas development}

\section{Analysis of transcription factors key for mouse pancreatic development establishes NKX2-2 and MNX1 mutations as causes of neonatal diabetes in man}

Flanagan SE, De Franco E, Lango Allen H, Zerah M, Abdul-Rasoul MM, Edge JA, Stewart H, Alamiri E, Hussain K, Wallis S, de Vries L, Rubio-Cabezas O, Houghton JA, Edghill EL, Patch AM, Ellard S, Hattersley AT

Institute of Biomedical and Clinical Science, University of Exeter Medical School, Exeter, UK

Cell Metab 2014;19:146-154

Background: Understanding transcriptional regulation of pancreatic development is required to advance current efforts in developing $\beta$-cell replacement therapies for patients with diabetes. Current knowledge of key transcriptional regulators has predominantly come from mouse studies, with rare, naturally occurring mutations establishing their relevance in man.

Methods: This study used a combination of homozygosity analysis and Sanger sequencing in 37 consanguineous patients with permanent neonatal diabetes to search for homozygous mutations in 29 transcription factor genes important for murine pancreatic development.

Results: The authors identified homozygous mutations in 7 different genes in 11 unrelated patients and show that NKX2-2 and MNX1 are etiological genes for neonatal diabetes, thus confirming their key role in development of the human pancreas. 
Conclusions: The similar phenotype of the patients with recessive mutations and mice with inactivation of a transcription factor gene support there being common steps critical for pancreatic development and validate the use of rodent models for $\beta$-cell development.

Pancreatic development is a complex process regulated by numerous transcription factors. Understanding the role of these transcriptional factors which regulate pancreatic development will be important for development of $\beta$-cell stem cells for the treatment of diabetes mellitus. Until recently, most of the knowledge about pancreatic development was derived from rodent models. However, research in the last few years is beginning to provide novel insights into human pancreatic development.

In this study the authors used a combination of homozygosity analysis and Sanger sequencing in 37 consanguineous patients with permanent neonatal diabetes to search for homozygous mutations in 29 transcription factor genes important for murine pancreatic development. They identified homozygous mutations in 7 different genes in 11 unrelated patients and show that the transcription factors NKX2-2 (NK2 transcription factor related, locus 2) and MNX1 (motor neuron and pancreas homeobox 1) are the cause of neonatal diabetes mellitus, thus confirming their key role in development of the human pancreas. Thus the transcription factors NKX2-2 and MNX1 play key roles in mice and human pancreatic development. This knowledge will help in the development of stem cell therapy for diabetes.

\section{Neonatal diabetes and developmental coordination disorder}

\section{Neuropsychological dysfunction and developmental defects associated with genetic changes in infants with neonatal diabetes mellitus: a prospective cohort study}

Busiah K, Drunat S, Vaivre-Douret L, Bonnefond A, Simon A, Flechtner I, Gérard B, Pouvreau N, Elie C, Nimri R, De Vries L, Tubiana-Rufi N, Metz C, Bertrand AM, Nivot-Adamiak S, de Kerdanet M, Stuckens C, Jennane $F$, Souchon PF, Le Tallec C, Désirée C, Pereira S, Dechaume A, Robert JJ, Phillip M, Scharfmann R, Czernichow P, Froguel P, Vaxillaire M, Polak M, Cavé H; French NDM Study Group INSERM U845, Université Paris Descartes, Sorbonne Paris Cité, Department of Paediatric Endocrinology, Gynaecology, and Diabetology, Necker-Enfants Malades Teaching Hospital, Assistance Publique-Hôpitaux de Paris, IMAGINE Affiliate, Paris, France

Lancet Diabetes Endocrinol 2013;1:199-207

Background: Neonatal diabetes mellitus is a rare genetic form of pancreatic $\beta$-cell dysfunction. The authors compared phenotypic features and clinical outcomes according to genetic subtypes in a cohort of patients diagnosed with neonatal diabetes mellitus before age 1 year, without $\beta$-cell autoimmunity and with normal pancreas morphology.

Methods: The authors prospectively investigated patients from 20 countries referred to the French Neonatal Diabetes Mellitus Study Group from 1995 to 2010. Patients with hyperglycaemia requiring treatment with insulin before age 1 year were eligible, provided that they had normal pancreatic morphology as assessed by ultrasonography and negative tests for $\beta$-cell autoimmunity. Changes were assessed in the 6q24 locus, KATP-channel subunit genes (ABCC8 and KCNJ11), and preproinsulin gene $(I N S)$ and investigated associations between genotype and phenotype, with special attention to extrapancreatic abnormalities.

Results: 174 index patients were tested, of whom $47(27 \%)$ had no detectable genetic defect. Of the remaining 127 index patients, $40(31 \%)$ had 6q24 abnormalities, $43(34 \%)$ had mutations in KCNJ11, 31 $(24 \%)$ had mutations in $A B C C 8$, and $13(10 \%)$ had mutations in INS. They reported developmental delay with or without epilepsy in 13 index patients (18\% of participants with mutations in genes encoding KATP channel subunits). In-depth neuropsychomotor investigations were done at median age 7 years (IQR 1-15) in 27 index patients with mutations in KATP channel subunit genes who did not have developmental delay or epilepsy. Developmental coordination disorder (particularly visual-spatial dyspraxia) or attention deficits were recorded in all index patients who had this testing. Compared with index patients who had mutations in KATP channel subunit genes, those with 6q24 abnormalities had specific 
features: developmental defects involving the heart, kidneys, or urinary tract [8/36 (22\%) vs. 2/71 (3\%); $\mathrm{p}=0.002$ ], intrauterine growth restriction [4/37 (92\%) vs. 34/70 (48\%); $\mathrm{p}<0.0001]$, and early diagnosis (median age 5.0 days, IQR 1.0-14.5 vs. 45.5 days, IQR 27.2-95.0; p < 0.0001). Remission of neonatal diabetes mellitus occurred in $89(51 \%)$ index patients at a median age of 17 weeks (IQR 9.5-39.0; median follow-up 4.7 years, IQR 1.5-12.8). Recurrence was common, with no difference between the groups who had 6q24 abnormalities versus mutations in KATP channel subunit genes ( $82 \mathrm{vs.} 86 \%$; $\mathrm{p}=0.36$ ).

Conclusions: Neonatal diabetes mellitus is often associated with neuropsychological dysfunction and developmental defects that are specific to the underlying genetic abnormality. A multidisciplinary assessment is therefore essential when patients are diagnosed. Features of neuropsychological dysfunction and developmental defects should be tested for in adults with a history of neonatal diabetes mellitus.

There has been an explosion of knowledge in the field of neonatal diabetes mellitus in the last 10 years. This study assessed the neuropsychological dysfunction and developmental defects in relation to the underlying genetic defect of the neonatal diabetes mellitus. Interestingly, those with neonatal diabetes mellitus due to $6 \mathrm{q} 24$ abnormalities had specific features such as developmental defects involving the heart, kidneys, or urinary tract and early diagnosis. Neuropsychological dysfunction was a common finding and the authors recommend a multidisciplinary team approach to these patients. Thus, patients with neonatal diabetes mellitus will require long-term follow-up for neuropsychological dysfunction and should be reviewed by a multidisciplinary team.

\section{How high-fat diet impairs hypothalamic melanocortin circuitry}

\section{Neonatal insulin action impairs hypothalamic neurocircuit formation in response to maternal high-fat feeding}

Vogt MC, Paeger L, Hess S, Steculorum SM, Awazawa M, Hampel B, Neupert S, Nicholls HT, Mauer J, Hausen AC Predel R, Kloppenburg P, Horvath TL, Brüning JC

Max Planck Institute for Neurological Research, Cologne, Department of Mouse Genetics and Metabolism, Institute for Genetics, University of Cologne, Excellence Cluster on Cellular Stress Responses in Aging Associated Diseases, and Center of Molecular Medicine Cologne, University of Cologne, Cologne, Germany

Cell 2014;156:495-509

Background: Maternal metabolic homeostasis exerts long-term effects on the offspring's health outcomes. Methods and Results: The authors demonstrate that maternal high-fat diet (HFD) feeding during lactation predisposes the offspring for obesity and impaired glucose homeostasis in mice, which is associated with an impairment of the hypothalamic melanocortin circuitry. Whereas the number and neuropeptide expression of anorexigenic proopiomelanocortin (POMC) and orexigenic agouti-related peptide (AgRP) neurons, electrophysiological properties of POMC neurons, and posttranslational processing of POMC remain unaffected in response to maternal HFD feeding during lactation, the formation of POMC and AgRP projections to hypothalamic target sites is severely impaired. Abrogating insulin action in POMC neurons of the offspring prevents altered POMC projections to the preautonomic paraventricular nucleus of the hypothalamus (PVH), pancreatic parasympathetic innervation, and impaired glucose-stimulated insulin secretion in response to maternal overnutrition.

Conclusion: These experiments reveal a critical timing, when altered maternal metabolism disrupts metabolic homeostasis in the offspring via impairing neuronal projections, and show that abnormal insulin signalling contributes to this effect.

The children of mothers with impaired metabolic homeostasis, as occurs in obesity, are metabolically programmed to have an increased risk of metabolic disorders. Here, the authors demonstrate that in mice, high-fat feeding during the critical phase of lactation reprograms neuronal pathways for metabolism with long-term implications for the offspring. The high-fat feeding triggers abnormal insulin signalling in the offspring that then alters hypothalamic neuronal circuits; it impairs the formation of melanocortin projections to preautonomic PVH. These neuronal circuits are critical in regulating metabolic pathways. It is interesting that abrogating POMC insulin action improves glucose 
metabolism despite maternal HFD. Thus, insulin signalling during lactation in the hypothalamic neuronal circuits seems to be critical in the long-term control of metabolism; the resulting abnormal insulin signaling in the offspring interferes with the formation of hypothalamic neural circuits that contribute to metabolic status.

\section{A novel therapy for severe hyperinsulinemic hypoglycemia}

\section{Sirolimus therapy in infants with severe hyperinsulinemic hypoglycemia}

Senniappan S, Alexandrescu S, Tatevian N, Shah P, Arya V, Flanagan S, Ellard S, Rampling D, Ashworth M, Brown RE, Hussain $\mathrm{K}$

Developmental Endocrinology Research Group, Clinical and Molecular Genetics Unit, Institute of Child Health, University College London, London, UK

N Engl J Med 2014;370:1131-1137

Background: Hyperinsulinemic hypoglycemia is the most common cause of severe, persistent neonatal hypoglycemia. The treatment of hyperinsulinemic hypoglycemia that is unresponsive to diazoxide is subtotal pancreatectomy.

Methods: The authors examined the effectiveness of the mammalian target of rapamycin (mTOR) inhibitor sirolimus in 4 infants with severe hyperinsulinemic hypoglycemia that had been unresponsive to maximal doses of diazoxide $(20 \mathrm{mg} / \mathrm{kg}$ b.w. per day) and octreotide $(35 \mu \mathrm{g} / \mathrm{kg}$ per day).

Results and Conclusions: All the patients had a clear glycemic response to sirolimus, although 1 patient required a small dose of octreotide to maintain normoglycemia. There were no major adverse events during 1 year of follow-up.

The congenital forms of hyperinsulinism lead to severe hypoglycaemia. The diffuse forms of congenital hyperinsulinism that are unresponsive to conventional medical therapy often require a near total pancreatectomy. However, a near total pancreatectomy will inevitably lead to lifelong diabetes mellitus and pancreatic exocrine insufficiency. There is thus an urgent need to develop novel medical therapies for patients with the diffuse form of congenital hyperinsulinism. In this proof of concept study the authors show that some patients with severe diffuse congenital hyperinsulinism may be treated with mTOR inhibitor drugs such as sirolimus and thus avoid the near total pancreatectomy. Four patients with severe hyperinsulinism were treated with sirolimus and all 4 showed a good glycemic response to this therapy. This is a promising beginning, however long-term follow-up is required in these patients given the side effects of mTOR inhibitors. Further research is required to understand the molecular basis of sirolimus action in these patients.

\section{How $\beta$-cell failure occurs in gestational diabetes}

\section{The furan fatty acid metabolite CMPF is elevated in diabetes and induces $\beta$-cell dysfunction}

Prentice KJ, Luu L, Allister EM, Liu Y, Jun LS, Sloop KW, Hardy AB, Wei L, Jia W, Fantus IG, Sweet DH, Sweeney G, Retnakaran R, Dai FF, Wheeler MB

Department of Physiology, University of Toronto, Toronto, ON, Canada

Cell Metab 2014;19:653-666

Background: Gestational diabetes (GDM) results from failure of the $\beta$ cells to adapt to increased metabolic demands. However, the cause of GDM and the extremely high rate of progression to type 2 diabetes (T2D) remains unknown.

Methods and Results: Using metabolomics, the authors show that the furan fatty acid metabolite 3-carboxy4-methyl-5-propyl-2-furanpropanoic acid (CMPF) is elevated in the plasma of humans with GDM, as 
well as impaired glucose-tolerant and T2D patients. In mice, diabetic levels of plasma CMPF induced glucose intolerance, impaired glucose-stimulated insulin secretion, and decreased glucose utilization. Mechanistically, CMPF acts directly on the $\beta$ cell, causing impaired mitochondrial function, decreasing glucose-induced ATP accumulation, and inducing oxidative stress, resulting in dysregulation of key transcription factors and ultimately reduced insulin biosynthesis. Importantly, specifically blocking its transport through OAT3 or antioxidant treatment could prevent CMPF-induced $\beta$-cell dysfunction. Conclusion: CMPF provides a link between $\beta$-cell dysfunction and GDM/T2D that could be targeted therapeutically.

The cause of $\beta$-cell exhaustion in type 2 diabetes mellitus and in gestational diabetes mellitus is not known. Using a metabolomics approach, furan fatty acid metabolite (3-carboxy-4-methyl-5-propyl-2furanpropanoic acid (CMPF)) was found to be raised in the plasma of humans with gestational diabetes and patients with type 2 diabetes. CMPF acts to inhibit mitochondrial function, induce oxidative stress and impair insulin synthesis. The translational implication is that by inhibiting CMPF one could potentially reduce $\beta$-cell dysfunction. Treatment with antioxidants has been proposed as an approach for the treatment of type 2 diabetes, and has been shown to attenuate islet fibrosis and apoptosis and improve glucose tolerance and insulin sensitivity in rodent models. The findings in this study show that pretreatment with the antioxidants can prevent CMPF from inducing $\beta$-cell failure, suggesting that antioxidant treatment may also be used in the prevention and/or treatment of gestational diabetes mellitus.

\section{B: Insulin growth factors in the neonatal period}

\section{Growth in preterm infants until six months post term: the role of insulin and IGF-I}

van de Lagemaat M, Rotteveel J, Heijboer AC, Lafeber HN, van Weissenbruch MM Pediatrics Department, VU University Medical Center, Amsterdam, The Netherlands Horm Res Paediatr 2013;80:92-99

Background: Since insulin-like growth factor type I (IGF-I) and insulin regulate growth in term infants, they were studied in relation to nutrient intake and growth until 6 months corrected age $(\mathrm{CA})$ in preterm infants.

Methods: In 138 preterm infants (51\% male, gestational age (expressed as median (IQR)) 30.6 (1.9) weeks, birth weight 1,368 (389) g) weight SDS, length SDS, IGF-I, and insulin were measured at term age, 3 and 6 months CA.

Results: IGF-I and insulin at term age were associated with weight SDS and length SDS at term age and 3 months CA. IGF-I and insulin at 3 months CA were associated with weight SDS and length SDS at 3 and 6 months CA. IGF-I and insulin at term age were negatively associated with gain in weight SDS and gain in length SDS between term age and 6 months CA (IGF-I: $\beta=-1.03,95 \%$ CI $-1.65,-0.41, p=0.001$ and $\beta=-0.78,95 \% \mathrm{CI}-1.32,-0.23, \mathrm{p}=0.005$; insulin: $\beta=-0.19,95 \% \mathrm{CI}-0.37,-0.01, \mathrm{p}=0.044$ and $\beta=-0.18,95 \% \mathrm{CI}-0.35,-0.01, \mathrm{p}=0.035)$. Nutrient intake was not associated with IGF-I or insulin.

Conclusion: The present study suggests that IGF-I and insulin are important growth regulators in preterm infants until 6 months CA, independent of nutrient intake.

Postnatal growth is mainly regulated by nutrients, insulin, and IGF-I. The sufficient supply of nutrients and adequate insulin action are important for fetal IGF-I production, indicating a linkage between growth and nutrient availability. In term infants, IGF-I levels are associated with postnatal growth and protein intake during infancy. In preterm infants, IGF-I levels are associated with protein intake until term age (40 weeks' postmenstrual age) and with postnatal growth until 6 months corrected age (CA). The present study was an observational analysis within a randomized controlled trial that evaluated the effects of post discharge formula, term formula, and human milk on growth and body composition of preterm infants. The authors found that IGF-I and insulin are important for growth in pre-term infants until 6 months of age CA independent of nutrient intake. This is another study which highlights the key role of IGF I in pre-term infants. 


\section{Preterm birth and subsequent insulin sensitivity: a systematic review}

Tinnion R, Gillone J, Cheetham T, Embleton N

Department of Neonatal Medicine, Royal Victoria Infirmary, Newcastle upon Tyne, UK

Arch Dis Child 2014;99:362-368

Background: The incidence of preterm birth is increasing worldwide. Evidence suggests that in later life these children are at increased risk of 'metabolic syndrome', which is itself associated with reduced insulin sensitivity (IS). The authors carried out a systematic review to examine whether preterm birth is associated with later changes in IS and whether a difference exists between those born small-for-gestational age (SGA) and appropriate-for-gestational age (AGA).

Methods: The authors used the Preferred Reporting Items for Systematic reviews and Meta-Analyses (PRISMA) guidance to structure the review with a priori data extraction criteria to answer the questions posed and then carried out the literature search. Only papers which included preterm infants in their study population and specifically assessed IS were included. Findings are reported by age group to enable change over the life course to be examined, even though the studies were mostly cross-sectional, observation studies.

Results: 26 suitable publications were identified and reviewed representing 20 separate cohorts, of which 16 had a term control group. The heterogeneity of the methods used to measure IS precluded metaanalysis. In infancy and early childhood there is a measurable association between IS and preterm birth. In later childhood and adulthood the strength of this association reduces, and current body composition becomes the variable most strongly associated with IS.

Conclusions: There is an association between preterm birth and IS throughout the life course, but the data are conflicting and associations are likely to be affected by the heterogeneity of each study population and multiple confounding factors that may change over time. While the optimal nutritional strategy for preterm infants remains to be determined, standard public health guidance to avoid obesogenic lifestyle factors remains equally important to individuals born preterm.

Low birth weight $(<2.5 \mathrm{~kg}$, LBW) is associated with reduced insulin sensitivity. Data also suggest that early-life growth during sensitive windows in the prenatal and postnatal period may programme later metabolic outcomes. Infants born preterm will generally be LBW. There are few data to distinguish between the effects of preterm birth, compared to LBW at term, on later metabolism, and preterm and term groups may have experienced in utero growth restriction (IUGR). The majority of published studies are epidemiological and focus on adults born small-for-gestational age (SGA, typically birth weight $<10$ th centile) who were born at term and were also LBW. This systematic review examined whether preterm birth is associated with later changes in insulin sensitivity and whether a difference exists between SGA and AGA infants. The authors used Preferred Reporting Items for Systematic reviews and Meta-Analyses (PRISMA) guidance for collecting and analyzing the data. They conclude that there is an association between preterm birth and insulin sensitivity throughout life but the data are not so clear. So the public health message is the avoidance of an obesogenic lifestyle in preterm infants.

\section{Administration of saccharin to neonatal mice influences body composition of adult males and reduces body weight of females}

Parlee SD, Simon BR, Scheller EL, Alejandro EU, Learman BS, Krishnan V, Bernal-Mizrachi E, MacDougald OA

Departments of Molecular and Integrative Physiology and Internal Medicine, and Division of Metabolism,

Endocrinology and Diabetes, School of Medicine, University of Michigan, Ann Arbor, MI, USA

Endocrinology 2014;155:1313-1326

Background: Nutritional or pharmacological perturbations during perinatal growth can cause persistent effects on the function of white adipose tissue, altering susceptibility to obesity later in life. Previous studies have established that saccharin, a non-nutritive sweetener, inhibits lipolysis in mature adipocytes and stimulates adipogenesis. This study tested whether neonatal exposure to saccharin via maternal lactation increased susceptibility of mice to diet-induced obesity. 
Methods and Results: Saccharin decreased body weight of female mice beginning post-natal week 3. Decreased liver weights on week 14 corroborated this diminished body weight. Initially, saccharin also reduced male mouse body weight. By week 5, weights transiently rebounded above controls and by week 14 male body weights did not differ. Body composition analysis revealed saccharin increased lean and decreased fat mass of male mice, the latter due to decreased adipocyte size and epididymal, perirenal and subcutaneous adipose weights. A mild improvement in glucose tolerance without a change in insulin sensitivity or secretion aligned with this leaner phenotype. Interestingly, micro-computed tomography $(\mu \mathrm{CT})$ analysis indicated saccharin also increased cortical and trabecular bone mass of male mice and modified cortical bone alone in female mice. A modest increase in circulating testosterone may contribute to the leaner phenotype in male mice.

Conclusions: This study established a developmental period in which saccharin at high concentrations reduces adiposity and increases lean and bone mass in male mice, while decreasing generalized growth in female mice.

Saccharin is an artificial sweetener with effectively no food energy. Artificial sweeteners have previously been shown to have uncharacterized metabolic effects on adipocyte differentiation and metabolism and that these effects of artificial sweeteners on adipose tissue biology may be largely independent of the classical sweet taste receptors, T1R2 and T1R3. The sweet taste receptors T1R2 and T1R3 are G protein-coupled receptors that function as carbohydrate sensors in taste buds, gut, and pancreas. It has previously been described that saccharin robustly induced adipogenesis in vitro. In contrast, the current study indicates administration of saccharin to neonatal mice slows female growth and reprograms male body composition. Although food intake and testosterone may play ancillary roles in the resulting phenotypes, the divergence between the in vitro and in vivo studies may result from the complex interplay of sweet and bitter taste receptor activation in multiple metabolic tissues. The fact that the male mice administered saccharin largely phenocopied mice deficient in sweet taste receptors points towards the possibility that saccharin is downregulating T1R2/T1R3 during development. In addition, one estimate suggests that $1-1.5 \%$ of adipocytes are turned over daily in rodents.

Thus, it is formally possible that saccharin increases adipogenesis, but an increased number of adipocytes is masked by an increased rate of adipocyte turnover. Future studies will need to clarify the role of sweet and bitter receptors in isolated cells and how they function together in vivo if the mechanisms used by saccharin to metabolically program mice are to be identified. Finally, long-term consequences of saccharin at clinical concentrations on lean, fat, bone, and glucose homeostasis will need to be determined.

\section{Insulin resistance and impaired pancreatic $\beta$-cell function in adult offspring of women with diabetes in pregnancy}

Kelstrup L, Damm P, Mathiesen ER, Hansen T, Vaag AA, Pedersen O, Clausen TD

Center for Pregnant Women with Diabetes, Department of Obstetrics, Research Unit 7821, Rigshospitalet,

Copenhagen University Hospital, Copenhagen, Denmark

J Clin Endocrinol Metab 2013;98:3793-3801

Background: Offspring of women with diabetes during pregnancy have an increased risk of glucose intolerance in adulthood, but the underlying mechanisms are unknown. The authors aimed to investigate the effects of intrauterine hyperglycemia on insulin secretion and action in adult offspring of mothers with diabetes.

Methods: A cohort of 587 Caucasian offspring, without known diabetes, was followed up at the age of 18-27 years. They included two groups exposed to maternal diabetes in utero: offspring of women with gestational diabetes mellitus $(n=167)$ or type 1 diabetes $(n=153)$. Two reference groups were included: offspring of women with risk factors for gestational diabetes mellitus but normoglycemia during pregnancy $(n=139)$ and offspring from the background population $(n=128)$. Indices of insulin sensitivity and insulin release were calculated using insulin and glucose values from a standard oral glucose tolerance test (120 min, 75 g glucose). Pancreatic $\beta$-cell function taking the prevailing insulin sensitivity into account was estimated by disposition indices.

Results: Both groups of offspring exposed during pregnancy to either maternal gestational diabetes or type 1 diabetes had reduced insulin sensitivity compared with offspring from the background popula 
tion (both $\mathrm{p}<0.005)$. The authors not find any significant difference in absolute measures of insulin release. However, the disposition index was significantly reduced in both the diabetes-exposed groups (both $\mathrm{p}<0.005$ ).

Conclusion: Reduced insulin sensitivity as well as impaired pancreatic $\beta$-cell function may contribute to the increased risk of glucose intolerance among adult offspring born to women with diabetes during pregnancy.

Maternal hyperglycemia during pregnancy induces lasting changes in the offspring. Intrauterine hyperglycemia is associated with an increased risk of overweight and the metabolic syndrome as well as glucose intolerance and overt type 2 diabetes in young adult Caucasian offspring of women with either gestational diabetes mellitus (GDM) or type 1 diabetes during pregnancy. The mechanisms that lead to glucose intolerance in infants born to mothers with pregnancy-induced diabetes mellitus are largely unknown. This is the first study to evaluate insulin sensitivity and insulin release in adult offspring of women with GDM. This study aimed to evaluate insulin sensitivity and insulin release in adult Caucasian offspring of women with either diet-treated GDM or type 1 diabetes during pregnancy compared with unexposed offspring. The main finding was that offspring exposed to hyperglycemia in utero, whether due to maternal GDM or type 1 diabetes, were characterized by impaired insulin sensitivity as well as a relatively reduced insulin release in comparison with offspring from the background population. The absolute level of insulin release did not significantly differ between exposed and non-exposed offspring.

\section{Growth patterns of large for gestational age infants}

\section{Growth patterns of large for gestational age children up to age $\mathbf{4}$ years}

Bocca-Tjeertes IF, Kerstjens JM, Reijneveld SA, Veldman K, Bos AF, de Winter AF

Division of Neonatology, Department of Pediatrics and Department of Health Sciences, University Medical Center

Groningen, University of Groningen, Groningen, The Netherlands

Pediatrics 2014;133:e643-e649

Background: This aimed to determine how growth of large for gestational age (LGA) preterm (PT) children was affected by their PT birth and their LGA status.

Methods: The authors performed a community-based cohort study of 1,302 PT and 489 full-term (FT) children, born 2002 and 2003.

Results: Growth in height, weight, and head circumference of LGA PT children was well balanced during infancy and only weight gain accelerated during subsequent years. This led to high BMIs comparable to those of LGA FT children. Being born both LGA and PT resulted in a median growth at the age of 4 years that was 0.1 SD lower for weight $(p=0.44), 0.1$ SD lower for height $(p=0.48)$, and 0.5 SD lower for head circumference compared with LGA FT counterparts $(p=0.016)$, whereas BMI at age 4 years was equal. Compared with appropriate for gestational age (AGA) PT children, these measures for LGA PT children were $0.9,0.6$, and 0.4 SD (all p < 0.001) higher, respectively. That led to the BMI of LGA PT children at age 4 years being significantly higher (0.9 points, i.e. 0.7 SD) than that of AGA PT and also higher $(0.6$ points, i.e. $0.4 \mathrm{SD})$ than that of AGA FT children.

Conclusion: The growth patterns of LGA PT-born children are distinctly different from other PT or FT children. In particular, they show substantially greater weight gains and relatively higher BMIs, which add to their already increased metabolic risks based on their gestational age.

Preterm (PT) and large for gestational age (LGA) birth (birth weight $>90$ th percentile) are associated with a negative pregnancy outcome. PT birth is a predictor of poor growth and long-term growth restriction in both early and moderately PT-born children. Small for gestational age (SGA) birth (birth weight $<10$ th percentile) as a proxy for intrauterine growth restriction is strongly associated with poor growth. Previous studies have shown that SGA infants remain smaller and LGA infants remain larger in size through early childhood, but the discrepancies in weight are primarily attributable to differences in lean body mass (muscularity). The aim of this study was to determine how growth was 
affected by PT birth and LGA status. A comparison was made of the absolute gains (number of $\mathrm{kg} / \mathrm{cm}$ attained per 1-year period and in total) of LGA PT children (LGA PTs) with three groups: appropriate for gestational age (AGA) PT children (AGA PTs), LGA FT children (LGA FTs), and AGA FT controls (AGA FTs). Then a comparison was made regarding age-standardized (relative) weight, height, head circumference $(\mathrm{HC})$, and BMI per age-year. Interestingly, the study found that the LGA PT infants gained weight more than those who were PT or full term.

\section{Accelerated infant weight gain and risk for non-alcoholic fatty liver disease in early adulthood}

Breij LM, Kerkhof GF, Hokken-Koelega AC

Department of Pediatrics, Subdivision of Endocrinology, Erasmus MC/Sophia Children's Hospital, Rotterdam, The Netherlands

J Clin Endocrinol Metab 2014;99:1189-1195

Introduction: Non-alcoholic fatty liver disease (NAFLD) is considered the hepatic metabolic syndrome. Some studies demonstrated an association between small size at birth and NAFLD. Rapid catch-up in weight often follows small birth size and has been associated with metabolic syndrome, but its association with NAFLD remained unknown.

Methods: In 268 adults aged 18-24 years, body mass index, waist circumference, triglyceride, $\gamma$-glutamyltransferase, alkaline phosphatase, alanine aminotransferase, and aspartate aminotransferase levels were determined. Fatty liver index (FLI; 0-100) was calculated. Associations of birth weight SD score and first year gain in weight and length SD scores were determined with FLI and other liver markers. Comparisons were performed between subjects with and without rapid catch-up in weight in the first year of life. Furthermore, a FLI score (low, intermediate, high risk for NAFLD) was assigned to each participant to determine clinical relevance, and ordinal regression analyses were performed.

Results: Gain in weight in the first 3 months of life was associated with FLI as a continuous variable, whereas low birth weight was not. There were no significant associations with $\gamma$-glutamyltransferase, alanine aminotransferase, or aspartate aminotransferase. Of the subjects with rapid catch-up in weight for length, $27.8 \%$ had an intermediate or high FLI at the age of 21 years, compared with $5.3 \%$ of subjects with slow catch-up. Rapid catch-up was also associated with a higher FLI score after adjustments (OR 11.7; $\mathrm{p}=0.016$ ).

Conclusion: Accelerated gain in weight for length in the first 3 months of life is associated with a higher risk for NAFLD in early adulthood, whereas small size at birth is not.

Non-alcoholic fatty liver disease (NAFLD) is a condition that resembles that of alcohol-induced liver injury, but it occurs in patients who do not abuse alcohol. NAFLD has the potential to progress to steatohepatitis, fibrosis, cirrhosis, and hepatocellular carcinoma. Here, the authors hypothesized that rapid gain in weight for length in the first 3 months of life, rather than LBW, might be associated with increased risk for developing NAFLD. Therefore they investigated the associations of birth weight, first-year gain in weight for length, and rapid catch-up in weight with the fatty liver index (FLI) at 21 years of age. A gain in weight SDS in the first 3 months of life was associated with a higher FLI at 21 years, whereas LBW SDS was not. Those subjects with rapid catch-up in weight for length had a significantly higher FLI in early adulthood than subjects with slow catch-up in weight, also after adjustment for birth weight SDS.

Formula-fed infants have a higher risk of being overweight in childhood and later in life. Infant feeding might thus influence later risk for NAFLD. Studies in rats showed that the liver might act as a systemic buffer, largely increasing its lipid content in the early stage of high-fat feeding. It has also been suggested that breast-feeding protects against the development of non-alcoholic steatohepatitis in children with NAFLD. This study did not have nutritional data to investigate the relationship between early nutrition, growth in infancy, and NAFLD risk factors in later life, but findings suggest that the use of nutrient-enriched formulas, which induce rapid weight gain in early life, might increase the risk for NAFLD later in life. 


\title{
Maternal factors associated with fetal growth and birth weight are independent determinants of placental weight and exhibit differential effects by fetal sex
}

\author{
Roland MC, Friis CM, Godang K, Bollerslev J, Haugen G, Henriksen T \\ Department of Obstetrics, Oslo University Hospital, Oslo, Norway \\ PLoS One 2014:9:e87303
}

Background: Maternal nutritional and metabolic factors influence the developmental environment of the fetus. Virtually any nutritional factor in the maternal blood has to pass the placental membranes to reach the fetal blood. Placental weight is a commonly used measure to summarize placental growth and function. Placental weight is an independent determinant of fetal growth and birth weight and modifies the associations between maternal metabolic factors and fetal growth. The authors hypothesized that maternal factors known to be related to fetal growth, newborn size and body composition are determinants of placental weight and that effects of maternal metabolic factors on placental weight differ between the genders.

Methods: The STORK study is a prospective longitudinal study including 1,031 healthy pregnant women of Scandinavian heritage with singleton pregnancies. Maternal determinants (parity, body mass index, gestational weight gain and fasting plasma glucose) of placental weight were explored by linear regression models, stratified by fetal sex.

Results: Parity, maternal BMI, gestational weight gain and fasting glucose had positive effects on placental weight. There was a sex-specific effect in these associations. Fasting glucose was significantly associated with placental weight in females but not in males.

Conclusion: Maternal factors known to influence fetal growth, birth weight and neonatal body composition are determinants of placental weight. The effect of maternal factors on placental weight is influenced by sex as illustrated in the relation between maternal glucose and placental weight.

Maternal nutritional and metabolic conditions (such as blood glucose) determine the environment in which the fetus develops. Maternal metabolic markers like plasma glucose and BMI are linearly related to birth weight, risk of macrosomia (birth weight $>90$ percentile) and percentage body fat of the newborn. Placental weight is a measure commonly used to summarize placental growth and aspects of placental function. In normal pregnancy it is reasonable to assume that placental weight is related to aspects of functional capacity of the placenta. In this large longitudinal cohort study, the authors found that parity, BMI, gestational weight gain and fasting glucose at visit 3 (weeks 30-32) and gestational age had positive, independent effects on placental weight. An increase of one unit of maternal $\mathrm{BMI}$ increased placental weight by $7.9 \mathrm{~g}$ (unadjusted). These findings are compatible with the concept that some of the effect of BMI on birth weight is mediated through a promotion of placental growth.

\section{Hyperinsulinemia stimulates angiogenesis of human fetoplacental endothelial cells: a possible role of insulin in placental hypervascularization in diabetes mellitus}

Lassance L, Miedl H, Absenger M, Diaz-Perez F, Lang U, Desoye G, Hiden U

Department of Obstetrics and Gynecology, Medical University of Graz, Graz, Austria

J Clin Endocrinol Metab 2013;98:E1438-E1447

Background: The insulin/IGF system regulates fetal and placental growth and development. In a pregnancy complicated by maternal diabetes, placentas are hypervascularized and fetal insulin levels are elevated. In the fetal circulation, insulin can act on the placenta through insulin receptors present on the fetoplacental endothelial cells. The authors hypothesized that insulin exerts proangiogenic effects on the fetoplacental endothelial cells, thereby contributing to the placental hypervascularization in diabetes.

Methods and Results: The effect of insulin on angiogenesis and proliferation of human fetoplacental endothelial cells was investigated by a two-dimensional network formation assay, staining for actin fibers, automatic cell counting, and cell cycle analysis. The signaling pathways involved were identified using antibodies against activated signaling proteins and pharmacological inhibitors. Insulin enhanced network formation by $23 \%(\mathrm{p}<0.05 \%)$ and caused actin reorganization. Insulin stimulated $(\mathrm{p}<0.05)$ 
phosphorylation of insulin receptor $(+320 \%)$, and insulin receptor substrate-1 $(+140 \%)$, Akt $(+177 \%)$, glycogen-synthase kinase- $\beta 3(+70 \%)$, and endothelial nitric oxide synthase (eNOS; $+100 \%)$ increased nitric oxide production and activated Ras-related C3 botulinum toxin substrate 1 (Rac1). Insulin did not induce ERK1/2 phosphorylation or proliferation. Inhibition of phosphatidylinositol 3-kinase, eNOS, and Rac1 signaling abolished the effects on network formation.

Conclusions: Elevated fetal insulin levels may contribute to the placental hypervascularization in diabetes via the phosphatidylinositol 3-kinase/Akt/eNOS pathway and involve Rac1. However, insulin does not stimulate proliferation and may need to cooperate with other growth factors.

The insulin/IGF system is required for normal fetal and placental growth and development. In pregnancies complicated by maternal diabetes mellitus, the placenta undergoes alterations in gene expression, morphology, and function. As a consequence of maternal and the ensuing fetal hyperglycaemia, fetal insulin levels increase. The fetoplacental vessels are in contact with the fetal circulation and thus can be affected by altered hormone and growth factor levels in fetal blood. This study investigated the effect of insulin on angiogenesis and proliferation of human fetoplacental endothelial cells. Insulin did not stimulate proliferation but might contribute to placental hypervascularization in diabetes via the phosphatidylinositol 3-kinase/Akt/eNOS pathway and involve Rac1. The main findings are that insulin stimulates in vitro network formation but does not induce proliferation, insulin-induced network formation involves the IRS1/PI3K/Akt signaling pathway and downstream eNOS and Rac1 activation, and insulin induces actin rearrangements involving PI3K, eNOS, and Rac1 signaling. These are the first results to show the proangiogenic effect of insulin on primary human fetoplacental ECs and to provide mechanistic evidence for insulin contributing to placental hypervascularization in maternal diabetes.

\section{Sleep disturbances are common in pregnancy}

\section{Interactions between pregnancy, obstructive sleep apnea, and gestational diabetes mellitus}

Reutrakul S, Zaidi N, Wroblewski K, Kay HH, Ismail M, Ehrmann DA, Van Cauter E

Section of Endocrinology, Department of Medicine, Rush University Medical Center, Chicago, IL, USA

J Clin Endocrinol Metab 2013;98:4195-4202

Background: Questionnaire studies have linked symptoms of obstructive sleep apnea (OSA) to the risk of gestational diabetes mellitus (GDM). Whether this association is present when OSA is assessed objectively by polysomnography is not known. This study aimed to assess the relationship between pregnancy, OSA, and GDM.

Methods: The authors conducted observational case-control studies using polysomnography in 15 nonpregnant, non-diabetic women (NP-NGT), 15 pregnant women with normal glucose tolerance (P-NGT), and 15 pregnant women with GDM (P-GDM). The groups were frequency matched for age and race/ ethnicity. Pregnant women were studied during the late second to early third trimester. Comparisons of OSA diagnosis and sleep parameters between NP-NGT and P-NGT were performed to assess the impact of pregnancy and between P-NGT and P-GDM to explore the association between GDM and OSA.

Results: Compared with NP-NGT, P-NGT women had a higher apnea hypopnea index (AHI) (median 2.0 vs. $0.5, p=0.03)$ and more disrupted sleep as reflected by a higher wake time after sleep onset (median 66 vs. $21 \mathrm{~min}, \mathrm{p}<0.01$ ) and a higher microarousal index (median 16.4 vs. 10.6, $\mathrm{p}=0.01$ ). Among the pregnant women, P-GDM had markedly lower total sleep time (median 397 vs. 464 min, p $=0.02$ ) and a higher AHI (median 8.2 vs. 2.0, $\mathrm{p}=0.05$ ) than P-NGT women. OSA was more prevalent in P-GDM than in P-NGT women ( 73 vs. $27 \%, \mathrm{p}=0.01$ ). After adjustment for pre-pregnancy body mass index, the diagnosis of GDM was associated with a diagnosis of OSA (OR 6.60; 95\% CI 1.1537.96). In pregnancy, after adjusting for pre-pregnancy body mass index, higher microarousal index significantly associated with higher hemoglobin $\mathrm{A}_{1 \mathrm{c}}$ and fasting glucose levels. Higher oxygen desaturation index was associated with higher fasting glucose levels.

Conclusion: Pregnancy is associated with sleep disturbances. Sleep is more disturbed in GDM than in P-NGT women. There is a strong association between GDM and OSA. 
During pregnancy, poor sleep quality and decreased sleep duration are common, even in the absence of obstructive sleep apnoea. Prospective studies show that symptoms of obstructive sleep apnoea increase during pregnancy, especially in women whose BMI exceeds $25 \mathrm{~kg} / \mathrm{m}^{2}$. Obstructive sleep apnoea in pregnancy is associated with preeclampsia, intrauterine growth retardation and preterm delivery. In this study, polysomnography was performed in 15 non-pregnant, non-diabetic women (NP-NGT), 15 pregnant women with normal glucose tolerance (P-NGT), and 15 pregnant women with GDM (P-GDM). Those women with GDM had more severe sleep disturbance then P-NGT with obstructive sleep apnoea being more common in the women with GDM. The possibility of obstructive sleep apnoea should be considered in pregnant women who are diagnosed with GDM, especially if other risk factors for obstructive sleep apnoea are present including obesity and hypertension. Conversely, for women with an established diagnosis of obstructive sleep apnoea who become pregnant, consideration should be given to early screening for GDM.

\section{E: Genome-wide association studies in neonatology}

\section{The chromosome 3q25 genomic region is associated with measures of adiposity in newborns in a multi-ethnic genome-wide association study}

Urbanek M, Hayes MG, Armstrong LL, Morrison J, Lowe LP, Badon SE, Scheftner D, Pluzhnikov A, Levine D, Laurie CC, McHugh C, Ackerman CM, Mirel DB, Doheny KF, Guo C, Scholtens DM, Dyer AR, Metzger BE, Reddy TE, Cox NJ, Lowe WL Jr; HAPO Study Cooperative Research Group

Division on Endocrinology, Metabolism and Molecular Medicine, Department of Medicine, University of Chicago, Chicago, IL, USA

Hum Mol Genet 2013;22:3583-3596

Background: Newborns characterized as large and small for gestational age are at risk for increased mortality and morbidity during the first year of life as well as for obesity and dysglycemia as children and adults. The intrauterine environment and fetal genes contribute to the fetal size at birth.

Methods: To define the genetic architecture underlying the newborn size, the authors performed a genome-wide association study in 4,281 newborns in four ethnic groups from the Hyperglycemia and Adverse Pregnancy Outcome Study. They tested for association with newborn anthropometric traits (birth length, head circumference, birth weight, percent fat mass and sum of skinfolds) and newborn metabolic traits (cord glucose and C-peptide) under three models: model 1 adjusted for field center, ancestry, neonatal gender, gestational age at delivery, parity, maternal age at oral glucose tolerance test (OGTT); model 2 adjusted for model 1 covariates, maternal body mass index (BMI) at OGTT, maternal height at OGTT, maternal mean arterial pressure at OGTT, maternal smoking and drinking, and model 3 adjusted for model 2 covariates, maternal glucose and C-peptide at OGTT.

Results: Strong evidence for association was observed with measures of newborn adiposity (sum of skinfolds model $3 \mathrm{Z}$-score $7.356, \mathrm{P}=1.90 \times 10^{-13}$, and to a lesser degree fat mass and birth weight) and a region on Chr3q25.31 mapping between CCNL and LEKR1. These findings were replicated in an independent cohort of 2,296 newborns. This region has previously been shown to be associated with birth weight in Europeans.

Conclusions: This study suggests that association of this locus with birth weight is secondary to an effect on fat as opposed to lean body mass.

The aim of this study was to identify genetic variants associated with measures of fetal size at birth. The authors performed a genome-wide-based association study looking at the genetic architecture of newborn size. The association study was performed on 4,281 newborns stratified into four ethnic groups. A highly significant association with the sum of skinfolds and, to a lesser extent, percent fat mass and birth weight at the 3q25.31 locus (which is intergenic between two genes, CCNL1 and LEKR1) was found. The mechanism by which the locus at 3q25.31 may impact fetal fat mass is not known. The nearest gene, CCNL1, encodes cyclin L1, a member of the cyclin family thought to be involved in the regulation of mRNA splicing as opposed to cell cycle control. 